\title{
The Role of Probability Distributions in Establishing Acceptance Sampling Plans and Quality Control Charts
}

\author{
Galal Abdulqader Ahmed Alashaari ${ }^{1}$, Fahad Sameer Alshammari ${ }^{2}$ \\ 1College of Science and Humanities, Prince Sattam bin Abdulaziz University, Saudi Arabia Email: \\ g.alashaari@psau.edu.sa \\ 2College of Science and Humanities, Prince Sattam bin Abdulaziz University, Saudi Arabia. Email: \\ f.alshammari@psau.edu.sa
}

Article History: Received: 10 November 2020; Revised 12 January 2021 Accepted: 27 January 2021; Published online: 5 April 2021

\begin{abstract}
This paper aims at identifying the probability distributions used in controlling the quality of products of industrial companies by examining the degree of the probability distributions' efficient in strengthen the control process that, consequently, would lead companies to cope with the new technologies to overcome the difficulties and problems they counter in controlling the quality of their products. This study used discrete and continuous probability distributions and explained how they can be used in acceptance inspection plans and quality control charts by examining the inputs and outputs of the production process and the production process itself. The results of this study indicated to the use of probability distributions maintains continuous improvement in quality and checks for convergence between inputs and outputs. In addition, the use of probability distributions enhances the efficiency and effectiveness of quality control. The results of this study are of importance to managements in the manufactured companies concerning the significance of using probability distributions to control the quality of products at all stages of production. Moreover, the establishment of periodical training programs specialized in quality control, with a focus on the use of probability distributions.
\end{abstract}

Keywords: Probability distributions, acceptance-sampling plans, quality control charts

\section{Background of the Study}

Probability distributions have proven its usefulness in the decision-making process. It meets the requirements for acceptance inspection by identifying and calculating the probabilities of acceptance for a production batch, it is examined for the purpose of acceptance or rejection. Or to inspect the production process for the purpose of conforming to the specifications; In order to continue production, modifying and reviewing process. Due to the importance of these distributions in controlling the quality of industrial products, the probabilities are used to determine the percentage of defective units produced under a stable production process. Each of the units that we select is considered an experiment, and the result of each experiment is determined by registering whether the unit is defective or not. That is, we use the defective ratio (non-matching) in the sample as an approximation of the probability of the appearance of a defective unit in the production process or the submitted sample for inspection. In this context, we are very interested in the random variable and the probability distribution, because they form the backbone of the Statistical inference. The probability distribution expresses the probabilities of all values that the random variable can be represented by X. It is expressed by mathematical formulas or probability functions that are used to determine the probabilities for each of the random variable values. regarding the difference in the sizes of populations (production batch - production line - continuous production ...) The probabilities calculated from these samples (the probability of acceptance) will change from one population to another. This leads to a difference in the samples selected from these populations. Therefore, each size of these populations has its own distribution for calculating these possibilities. (Dradkeh 2001: 95). That is, the probability distributions play a role in quality control, and that's when we want to estimate the percentage of the samples which has a particular characteristic such as the defectiveness percentage of the submitted sample or the production process. By determining the appropriate sample size, the type of sampling that it is supposed to be, the form of selecting the sample. As they are essential conditions for the use of probability distributions in quality control. Probability theory categorizes distributions into discrete distribution and continuous distribution. Discrete distributions are used to calculate the probabilities of a number of units that do not conform to specifications (defective) in a 
sample, or in the statistical population, and is used to count the defective units or the number of defectives in one piece. Among the most important of these distributions used in quality control are: Hypergeometric Distribution. Binomial Distribution. - Poisson Distribution- Normal distribution.

The main problem that the research seeks to study is determined by the lack of many Arab industrial companies to use the probability distributions in examining the inputs and outputs of the production process and during the course of the production process. In this research, we seek to identify the probability distributions and how to use them in the various stages of their production processes in order to rise to the distinctive level in production. The importance of this research is that many Arab newly existed industries face strong competition from foreign products. The reason behind this is the abundant number of defectives and defects found in our industrial products. Therefore, these companies must develop, improve and control the quality of their goods. And support them with statistical techniques that achieve improving the quality and help them to face intense global competition. Therefore, the aim of this research is to introduce probabilities distributions and indicate how they can be used to control the quality of the industrial products at every stage of production to address and solve the problems they face.

\section{Research methodology}

In preparing this research, the researcher adopted the following two approaches:

1-The deductive approach: through the theoretical study of research, based on a review of the statistical literature on statistical sampling techniques, and methods of statistical quality control, and by making use of the recent academic proposals contained in previous books and studies, Arab and foreign periodicals and bulletins, and on what was obtained from the Internet, and other official documents of the two companies.

2-The inductive approach: by using practical examples through the use of data on the reality of actual production.

\section{Theoretical part}

\subsection{Hyper geometric Distribution}

This distribution is regarded as one of the most important discrete potential distributions and plays a major role in the field of quality control. In general, in matters related to the ending (limited) statistical population from which the random sample is taken without return. The equation for this distribution is extracted using simple counting (combinations) techniques.

Probability function:

$$
P(r ; N, M, n)= \begin{cases}\frac{C_{r}^{M} C_{n-r}^{N-M}}{C_{n}^{N}} ; & \max (0, n-N+M) \leq r \leq \min (n, M) \\ 0 & \text { Otherwise }\end{cases}
$$

Whereas: $\mathrm{N}, \mathrm{M}, \mathrm{n}$ are the distribution parameters and all are positive numbers; It must be identified. $\boldsymbol{M} \leq \boldsymbol{N}, \boldsymbol{n} \leq \boldsymbol{N}$

, $\mathrm{P}(\mathrm{r})$ The probability of finding a defective single (r) from a random sample size (n) unit. It was chosen without returning from a production batch of single $(\mathrm{N})$ size, including a defective single $(\mathrm{M})$.

$\mathrm{N}$ the number of units in a batch.

$\mathrm{n} \quad$ the number of units in the sample.

$\mathrm{M}$ the number of non- matching units in a batch.

$\mathrm{r}$ the number of non- matching units in the sample.

$\mathrm{N}-\mathrm{M}$ the number of matching units in a batch.

$n-r$ the number of matching units in the sample.

This distribution has a cumulative function (Distribution Function) which gives a greater or less probability than a number of non-matching units (r) in a sample size (n.) This function is given as follows:

$$
F(c)=P(r \leq c ; N, M, n)=\sum_{r=0}^{c} \frac{C_{r}^{M} C_{n-r}^{N-M}}{C_{n}^{N}} \ldots \ldots \ldots .
$$

Where $\mathrm{c}$ : the maximum number of unmatched units (acceptance number).

The mean is 
$E(c)=\frac{n M}{N}$

Its variance equals

$$
\operatorname{var}(c)=\frac{n M}{N} \cdot \frac{N-M}{N} \cdot \frac{N-n}{N-1}=n p q \frac{N-n}{N-1}
$$

One of the advantages of this distribution is that, if the number of non-matching units is known, selecting with returning is not necessary to get correct results. As for the disadvantages of using it in the field of industry is that, the statistical population are often large, and that the exact number of unmatched units is unknown. Likewise, the calculations are difficult even when using the statistical calculator that gives the compatibility directly. (Robert, 2002: 215)., Therefore, this distribution can be applied in the field of quality control, if the following conditions are met: (Weimer, 1993: 261).

1- The production contains the matching units and the non-matching units.

2- The production batch should be limited.

3- To choose the sample randomly.

4- The selecting should be without returning.

Robert. J Pond adds. is a fifth condition; That is, the sample size must be greater than $10 \%$ of the batch size, that is, $\mathrm{n} / \mathrm{N}>0.10$. (Robert, 2002: 215).

\subsection{Binomial Distribution}

The binomial distribution is used in the quality inspection to estimate a percentage in a quality control population. It is part of the units of this community (p) that have special characteristics (damaged - defective not matching ), and other units (1-p) do not have such characteristics. Such a population is called a binomial population, for example when a production unit is selected randomly from the production process; or from the production line; Or from a certain production batch submitted for inspection. Either this unit is defective (nonmatching with specifications) or is intact (matching with specifications). When this option is repeated (n) once under the same circumstances and the selecting with the returning, the results of this selecting are independent. The outcome of the experiment is either defective or intact; so the random variable in this case that we are interested in is the number of defective units which we observe from among the sample units whose size is (n) single. The possible values taken by the random variable are $0,1,2, \ldots,(\mathrm{n})$ and the random variable $(X)$, which represents the number of successes, follows the binomial distribution when the experiment consists of (n) an identical and independent attempt. The result of each attempt consists of either success (defective) or failure (intact). And that the probability of success be constant does not change from one attempt to another. That the inspection be with returning; This is the basic concept of this distribution. That is, the inspection with the returning confirms that the probability of success (p) or failure (1-p) is constant from one attempt to another. Therefore, random selection confirms independence, so, inspection with returning confirms the binomial distribution that the probability of success (p) remains constant from one attempt to another. The attempts (n) are independent of each other; but in practical applications, we find that random sampling takes place without returning as a result of the large sizes of these populations, to the extent that the change in the probability of randomly selecting from one to the other is not significant. This does not contradict the application of binomial in the case of large population, and the selecting takes place without returning (George, 2004: 200). And the role that the binomial distribution plays, which is to estimate the defective rate, any defects are identified in this distribution. The probability of obtaining (r) defective units in the sample size (n) using a binomial distribution is calculated by the following probability mass function:

$$
P(r ; n, p)=\left\{\begin{array}{lr}
C_{r}^{n}(p)^{r}(q)^{n-r} & , r=0,1,2, \ldots, n \\
0 & \text { Otherwise }
\end{array}\right.
$$

two distribution parameters, $\mathrm{n}$ is a positive and positive number (number of attempts). \& $0 \leq p \leq 1$ and the accumulative function for this distribution is

$$
F(r)=p(r \leq c ; n, p)=\sum_{r=0}^{c} C_{r}^{n} \cdot p^{r} \cdot(1-p)^{n-r}
$$

There are tables for this distribution which show the cumulative probability of any of the values of the variable $(r)$, It has different values for $(p, n)$. The mean values, variance and standard deviation for this distribution are given as follows:

$$
E(r)=n \cdot p
$$




$$
\begin{aligned}
& \Rightarrow E\left(\frac{r}{n}\right)=p \\
& \sigma_{n p}^{2}=\operatorname{var}(r)=n p(1-p) \\
& \sqrt{\operatorname{var}(r)}=\sqrt{n p(1-p)}
\end{aligned}
$$

It is very important to distinguish between the average number of the taken place attempts in (n) experiment, which is (np); and the probability of the attempt (p.). That is, to distinguish between the mean number of units rejected in the samples; And the average percentage rejected. The mean percentage rejected in any sample is the number of rejected units divided by the sample size (n.). The standard deviation of the rejected ratio is the standard deviation of the number of rejected units $(\sqrt{n p(1-p)})$ divided by the sample size (n), that is:

$$
\sigma_{p}=\sqrt{n p(1-p)} / n=\sqrt{\frac{p(1-p)}{n}}
$$

The binomial distribution is used in the case of independent or assumed independent incidents. It is also used in the absence of the assumption of independence. This is really what we do in most quality control work, and this is due to the assumption of the appropriate size of population to accept the imposition of independence; As there is an important statistical fact, which is: it enables us to assume independence in the event that small samples are selected from a large number of units of population without doing anything may end up wrong. This fact is taken in some discrete distributions, including binomial ones, in summary, if the sample size (n) is less than $10 \%$ of the population size, for example ( $\mathrm{n} / \mathrm{N}<0.1$ ), then the binomial converges with the Hypergeometric. We notice from the binomial distribution compared to the Hypergeometric distribution, it is not necessary to identify the size of the population or the number of defective units in the population; because it is not used in the calculation. However, the binomial distribution has disadvantages: (Robert, 2002: 221):

1- Assuming that the percentage of defective units remain constant.

2- Independence can be assumed only if the sizes of societies are large, and this is often the case in permanent, stable production.

3- Despite the easiness of calculations, they are tedious compared to the Hypergeometric.

Finally, in order to implement this distribution in accordance with the concept of quality control; The following conditions must be fulfilled (Gardner, 1993: 14):

1. Each inspection unit shall be classified as accepted or rejected.

2. selecting the sample randomly.

3. The probability of obtaining a non-matching unit is constant.

4. The result of the inspecting any unit produced is independent from the other, that is, it is not affected by the result of inspecting any other unit.

\subsection{Poisson distribution}

This distribution is often known as the law of small numbers and is sometimes called the distribution of rare incidents. It is very useful in our study here (quality control). Especially, in studying the percentage of nonmatching units when this percentage is less than 15\%. It is the most used in the inspection of quality control and its plans. This distribution is used in the following cases: (Alsa'ed, 2000: 223):

1. The probabilities of defects are few.

2. The sample size is small, when compared to the size of the population, it is considered small.

The number of community units is large.

This distribution is used in preparing quality control charts for samples; calculating the probability upon acceptance inspection plans. For this very distribution, it is not possible to obtain the results and calculations of individual and collective probabilities for all values (np), where (n) is large; whereas, (q \& p) are small, so (np) is small (np <5).

The probability function of this distribution is:

$$
p(r ; \lambda)= \begin{cases}\frac{\lambda^{r} \cdot \lambda^{-\lambda}}{r !} & ; r=0,1,2, \ldots \\ 0 & \text { Otherwise }\end{cases}
$$

Where: $0>\mathrm{np}=\lambda$, which is the parameter of this distribution. $\mathrm{C}$ represents the number of matching $\mathrm{s}$ in the sample for a given classification 
$\lambda$ : represents the mean number of mismatches in the sample for a given classification.

We notice from the previous figure the effect of $\lambda$, where, the single possibilities of the Poisson distribution decrease rapidly with increasing values of the random variable (r), This results in the Poisson distribution always being warped for any value of $\lambda$. This results in the Poisson distribution always having a torsion of any value for $\lambda$, and as shown in the previous figure in which the torsion appears during the three cases. However, the torsion decreases as the value $\lambda$ increases.

$$
\begin{aligned}
& \text { And so it is } r \approx p(\lambda) \\
& \text { With a mean } E(r)=\lambda=n p \\
& \text { And variance } \operatorname{var}(r)=n p \\
& F(r)=p(r \leq c ; n p)=e^{-\lambda} \sum_{r=0}^{c} \frac{\lambda^{r}}{r !} \text { where } \lambda=n p
\end{aligned}
$$

The Poisson distribution is used as an approximation to the binomial distribution if the population size is large. That is, if there is an abundant amount of production or there is permanent stable production, the percentage of the discordant units was less than 0.1, This distribution has tables, and because of these tables, it is preferable to approximate the binomial with a Poisson distribution. This corresponds to a great extent with most industrial studies. Since one of the most important applications of Poisson distribution in industrial studies is to have an idea about the quantity of defective units, or units which need to be reprocessed as a result of setting limits to the number of discordant prior to the scrapping or reprocessing (Robert, 2002: 225). According to the concept of quality control, we summarize the conditions for applying the Poisson distribution as follows: (Gardner, 1993: 16).

Identifying the inspected unit is constant with time.

The unit is either matching to or not matching to the specifications (accepted or rejected).

Too many opportunities for defects to exist in each unit.

The possibility of non-matching (defective) anywhere in each unit is very small.

The probability is constant, that is, $\mathrm{p},(1-\mathrm{p})$ constant.

\subsection{Normal Distribution}

This distribution is regarded as one of the most important continuous probability distributions and the most used in the field of industry and quality control and depends on random samples when using the statistical inference. Many phenomena follow or are approaching this distribution, perhaps one of the most prominent reasons that made this distribution unique to this distinguished site is that, this distribution can be considered as the central distribution to which all probability distributions (continuous and discrete) belong. This is confirmed by the central end theory.

Among the phenomena which follow the normal distribution; The big role this distribution plays in studying the variables and specifications that are used in the field of quality control.

Therefore, most of the practical experiences and statistical studies have proven that the nature of deviations which exist in the production process take the form of a bell when represented graphically. This is what we will see in the control maps, and its ends extend endlessly (Bishmani, 2000: 37), and this distribution is useful in determining the probabilities of the results of an event in the future.

The normal distribution has a function of its probable density:

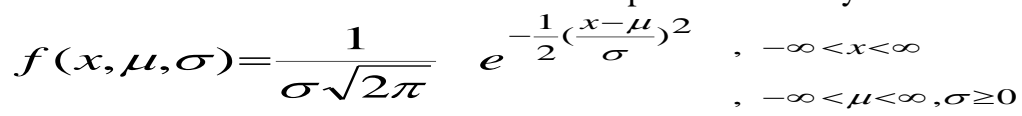

Where $\mathrm{x}$ : the individual value,: $\mu, \sigma$ distribution parameters.

$$
\operatorname{var}(X)=\sigma^{2}
$$

The mean is $\boldsymbol{E}(\boldsymbol{X})=\boldsymbol{\mu}$ and the variance is

We use the probability of having $99.74 \%$ of the measurements between the three standard deviations from the mean when finding the control diagram. 


\section{Distribution relationship}

In many probability distributions, it is sometimes difficult to identify when to apply these distributions. Accordingly, we will perform a simple review in which we show the process of convergence between these distributions as approximate indicative cases and not as absolute laws. As this approximation helps to save time and effort in calculations, especially in cases when it is not possible to use the schedules of one of the distributions.

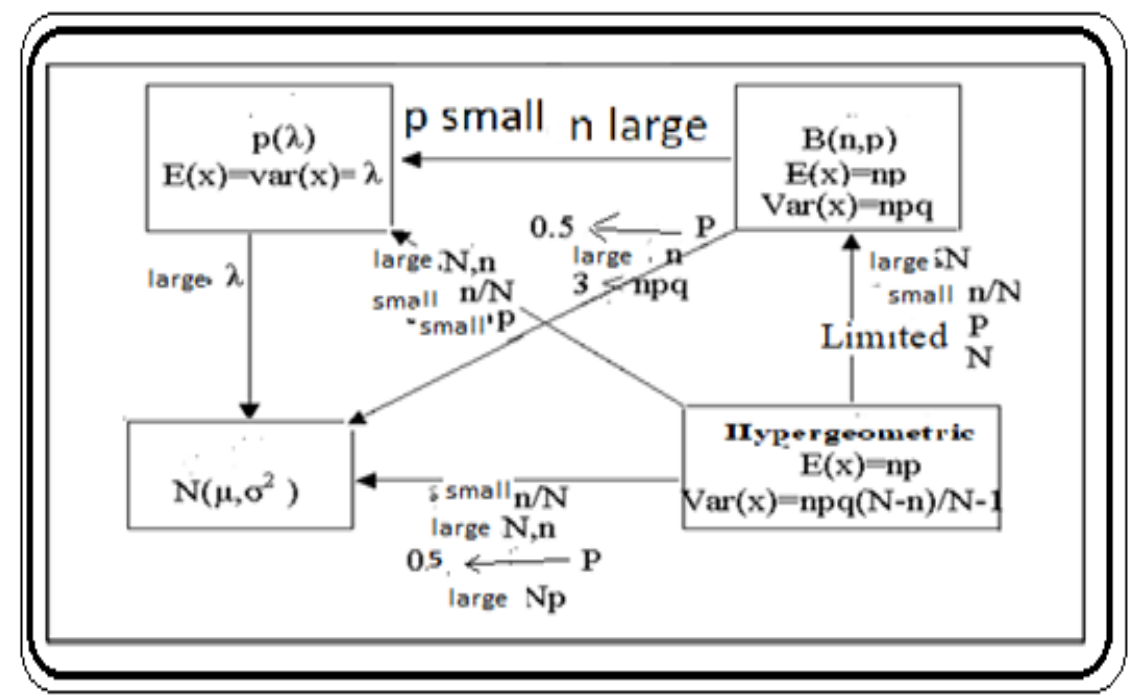

Figure 1. shows the convergence of distributions towards each other: Source: Researcher

From the above mentioned, we conclude that when conducting an inspection of an industrial product by calculating the various probabilities by the above mentioned probability distributions. It is a must to identify these distributions accurately. This accuracy is determined by statistical sampling techniques through the type, shape and size of the sample. We also realized the importance and usefulness of these distributions, which proved to be very useful in the decision-making process, which will benefit us to meet the acceptance inspection needs, control panels by specifying and calculating the acceptance probabilities of a production batch, inspecting for the purpose of acceptance or rejection or for inspecting the production process for the purpose of matching the characteristics in order to continue production or modifying and reviewing of this process. We also notice that there are no specific rules which indicate when to use Approximation methods. On one hand, the budgets of the accounts and time saved must be balanced. And the possible mistake made when using this approximation, on the other hand. As experience also plays a major role in the approximation process, which helps to save time and effort in calculations.

\section{Previous Studies and Hypothesis}

Al-Ariqi study 2002, "The effect of applying quality standards on marketing performance" (applied study at AlBarh Cement Factory in Yemen). The study sought to achieve many goals, the most important of which is determining the level of application of the approved quality standards in Al-Barah Cement Factory, as well as the reality of the quality of productive performance in the factory. The study used the method of quality control maps for the variables for the mean and range. Through the use of actual data for the production process of calcic saturation factor. In this aspect, the study found that the productive process of calcic saturation factor is under the control of statistical quality and within the limits of the specific specification. The study also used quality control maps, as quality control maps were used to inspect the number of defects (C-Chart). The tested characteristic was the number of defectives in the packaging of the cement bags (torn bags), which appear during the packaging process for the cement product. The results indicated that the production process (packaging process) is unstable and most of it falls outside the boundaries of quality control, which indicates that there are non-random, diagnostic reasons that must be identified and corrected.

Hongyi Sun 2000 study, TQM, ISO 9000 "certification and performance improvement".The study indicated that companies holding an ISO certificate achieve better than non-obtaining companies in terms of reducing customer complaints, increasing the degree of matching of products to pre-determined specifications and improving productivity, and increase production, as the study indicated that the ISO certificate has a positive impact on the application of total quality management. 
Peter Knutron's 1994 study, "A model approach to self-assessment." The study indicated that organizations which implement total quality management achieve a global competitive advantage and create a manufacturing environment that achieves customers' satisfaction and continuous and improvement become a part of its culture. The study also indicated that the company under study (the pump industry) is using the European model to achieve continuous improvement in its operations. The company also started implementing a supportive change program that included the following:

- A series of sessions to solve problems by hiring an external expert.

- Forming work teams. using measurement techniques. monitor consumers' satisfaction.

From the above mentioned, it is clear from the previous studies that most of its attention focused on controlling production quality in its second stage only (the production process during operation) without exposure to controlling the production quality in the input and output stages. As for our research, we have focused on making this research to acquire the characteristic of distinction, renewal, and avoiding repetition. This is done through addressing the issue of statistical control of quality in an integrated manner in a way that enables us to control the product in all its production stages.

The research seeks to test the following hypothesis:

The use of probability distributions at all stages of the production process raises the efficiency and effectiveness of quality control.

\section{Research results}

Probability distributions were used to control the quality of the inputs and outputs of the production process when designing sampling plans according to the product condition or the consumer requirement, or both. Probability distributions are used to calculate the different acceptance probabilities, and to create OC curves for each plan, with the help of the statistical program from Min-tab or any other program in performing calculations of acceptance probabilities, and implementing graphics and graphs in designing the specifications acceptance inspection based on consumer risk (consumer condition).

According to the following data:

600 can $=, \mathrm{Ac}=0, \mathrm{Re}=1, \mathrm{can} \mathrm{n}=46,=0.10=\mathrm{Pa}, \quad \beta \mathrm{LQL}=\mathrm{P} 0.10=0.05$

To show the chances of accepting the batch at a certain quality level of incoming, we draw the OC curve representing this plan as in Figure 1. Where we have identified 11 points for the proportions of defective (nonmatching) units that represent the quality inserted, which was satisfied to draw the OC curve, and calculates the corresponding acceptance probabilities of these points with a binomial distribution, using the Minitab program.

The results of the acceptance calculations were as follows:

MTB > CDF 0;

SUBC> Binomial 460.001 .

Cumulative Distribution Function

Binomial with $\mathrm{n}=46$ and $\mathrm{p}=0.00100000$

$\mathrm{x} \quad \mathrm{P}(\mathrm{X}<=\mathrm{x})$

$0.00 \quad 0.9550$

MTB > CDF 0;

SUBC> Binomial 460.004 .

Cumulative Distribution Function

Binomial with $\mathrm{n}=46$ and $\mathrm{p}=0.00400000$

$\mathrm{x} \quad \mathrm{P}(\mathrm{X}<=\mathrm{x})$

$0.00 \quad 0.8316$

$\mathrm{MTB}>\mathrm{CDF} 0$;

SUBC> Binomial 460.005 .

Cumulative Distribution Function

Binomial with $\mathrm{n}=46$ and $\mathrm{p}=0.00500000$

$\mathrm{x} \quad \mathrm{P}(\mathrm{X}<=\mathrm{x})$

$0.00 \quad 0.7941$

MTB > CDF 0;

SUBC> Binomial 460.008.

Cumulative Distribution Function 
Binomial with $\mathrm{n}=46$ and $\mathrm{p}=0.00800000$

$\mathrm{X} \quad \mathrm{P}(\mathrm{X}<=\mathrm{x})$

$0.00 \quad 0.6911$

MTB > CDF 0;

SUBC> Binomial 460.01.

Cumulative Distribution Function

Binomial with $\mathrm{n}=46$ and $\mathrm{p}=0.0100000$

$\mathrm{x} \quad \mathrm{P}(\mathrm{X}<=\mathrm{x})$

$0.00 \quad 0.6298$

MTB > CDF 0;

SUBC> Binomial 460.02 .

Cumulative Distribution Function

Binomial with $\mathrm{n}=46$ and $\mathrm{p}=0.0200000$

$\mathrm{X} \quad \mathrm{P}(\mathrm{X}<=\mathrm{x})$

$0.00 \quad 0.3948$

MTB > CDF 0;

SUBC> Binomial 460.03.

Cumulative Distribution Function

Binomial with $\mathrm{n}=46$ and $\mathrm{p}=0.0300000$

$\mathrm{X} \quad \mathrm{P}(\mathrm{X}<=\mathrm{x})$

$0.00 \quad 0.2463$

MTB > CDF 0;

SUBC> Binomial 460.04 .

Cumulative Distribution Function

Binomial with $\mathrm{n}=46$ and $\mathrm{p}=0.0400000$

$\mathrm{X} \quad \mathrm{P}(\mathrm{X}<=\mathrm{x})$

$0.00 \quad 0.1529$

MTB > CDF 0;

SUBC> Binomial 460.05 .

Cumulative Distribution Function

Binomial with $\mathrm{n}=46$ and $\mathrm{p}=0.0500000$

$\mathrm{X} \quad \mathrm{P}(\mathrm{X}<=\mathrm{x})$

$0.00 \quad 0.0945$

MTB > CDF 0;

SUBC> Binomial 460.06 .

Cumulative Distribution Function

Binomial with $\mathrm{n}=46$ and $\mathrm{p}=0.0600000$

$\mathrm{X} \quad \mathrm{P}(\mathrm{X}<=\mathrm{X})$

$0.00 \quad 0.0581$

MTB > CDF 0;

SUBC> Binomial 460.07 .

Cumulative Distribution Function

Binomial with $\mathrm{n}=46$ and $\mathrm{p}=0.0700000$

$\mathrm{x} \quad \mathrm{P}(\mathrm{X}<=\mathrm{x})$

$0.00 \quad 0.0355$

Table (1) shows the acceptance probabilities corresponding to the quality inserting the first acceptance inspection plan at $\mathrm{Ac}=0$.

- The second acceptance inspection plan at the acceptance number Ac $=1$

600 can $=\mathrm{N}, \mathrm{Ac}=1, \mathrm{Re}=2$ can $\mathrm{n}=78,=0.10=\mathrm{Pa}, \boldsymbol{\beta L Q L}=\mathrm{P} 0.10=0.05$

We draw the OC curve representing this plan, as shown in Figure 1. At the same points for the ratios of the defective (non-matching) units that represent the quality entered, and to calculate the acceptability possibilities corresponding to those points shown in Table 1, which was calculated in the same way as the previous method used in the first plan using Minitab.

- Third acceptance inspection plan at $\mathrm{Ac}=2$

600 can $=\mathrm{N}, \mathrm{Ac}=23, \mathrm{Re}=$, $\mathrm{can} \mathrm{n}=106,=0.10=\mathrm{Pa}, \boldsymbol{\beta L Q L}=\mathrm{P} 0.10=0.05$

And Figure No. (1) shows the OC curves of this plan at the same points for the ratios of the defective units that represent the quality inserted, and the possibilities of acceptance are shown in Table No. (1). 
Table 1. The acceptance possibilities of $\mathrm{Pa}$ at the quality values inserted $\mathrm{P} 0$ for the three plans, and at the different Ac values

\begin{tabular}{|c|c|c|c|c|c|c|c|c|c|c|c|c|}
\hline \multicolumn{13}{|l|}{$100 p$} \\
\hline & & 0.1 & 0.4 & 0.5 & 0.8 & 1 & 2 & 3 & 4 & 5 & 6 & 7 \\
\hline \multicolumn{13}{|l|}{-1} \\
\hline $\mathrm{AC}=0$ & $\mathrm{~Pa}$ & 0.955 & 0.8316 & 0.7941 & 0.6911 & 0.6298 & 0.3948 & 0.2463 & 0.1529 & 0.0945 & 0.0581 & 0.0355 \\
\hline \multicolumn{13}{|l|}{$\mathrm{n}=46$} \\
\hline \multicolumn{13}{|l|}{-2} \\
\hline $\mathrm{AC}=1$ & $\mathrm{~Pa}$ & 0.971 & 0.9607 & 0.9415 & 0.8706 & 0.8164 & 0.5361 & 0.3171 & 0.176 & 0.0634 & 0.0475 & 0.0239 \\
\hline \multicolumn{13}{|l|}{$\begin{array}{c}\mathrm{n}= \\
78\end{array}$} \\
\hline \multicolumn{13}{|l|}{-3} \\
\hline $\mathrm{AC}=2$ & $\mathrm{pa}$ & 0.9998 & 0.9909 & 0.9835 & 0.9461 & 0.9093 & 0.6439 & 0.3803 & 0.1991 & 0.0957 & 0.0432 & 0.0185 \\
\hline
\end{tabular}

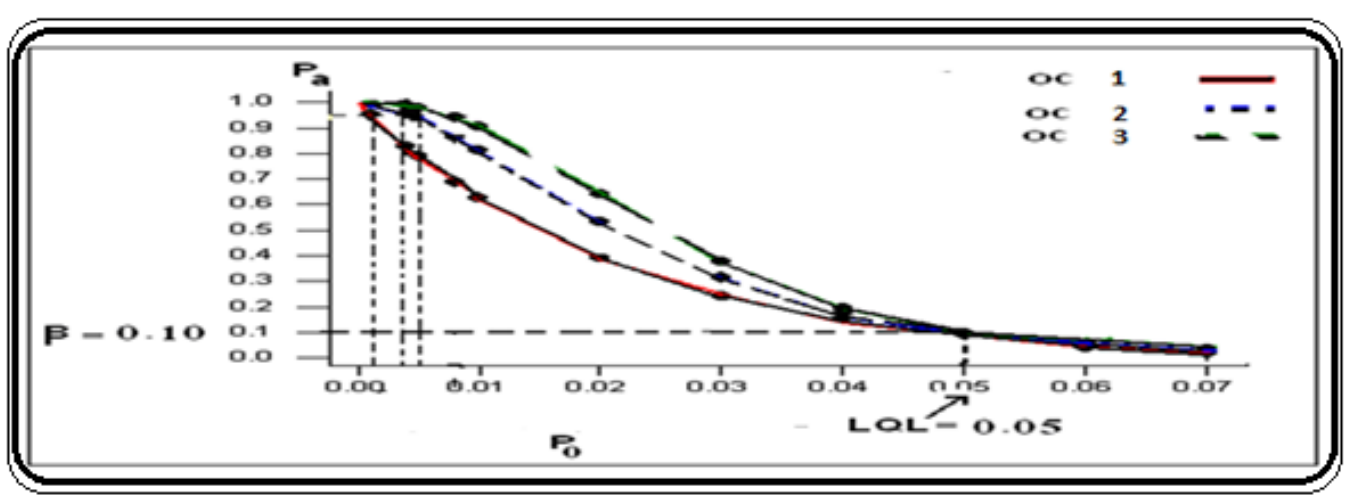

Figure 2. The OC curves for three plans at $\mathrm{Ac}=0,1,2$

Using the normal distribution to check and control quality in the second stage of production. It is the stage of the production process (inspection during operation), to ensure that the required quality level is maintained. And work on the continuous improvement of these products, by studying the behavior of the production process represented by studying the differences between the production units during the operation of the process, explain its causes and treatment, using statistical sampling techniques by selecting periodic random samples to distinguish changes in the process over time (according to the differences which appear between the samples), and by drawing the so-called quality control panels, which gives their regular use a strong foundation to evaluate the stability of the parameters of the production process in tracking a specific statistic path on the control panels.

From the above mentioned, we find that the quality control panels depend on their formation on the periodic random samples selected from the production process, which produces the values of the statistics of interest, and we will explain in our applied study here when creating the various quality control panels where practical experiences have proven that deviations in the production process follow the normal distribution in the case of certain conditions as explained by Schwart (General Secretariat of the Arab Organization for Standardization and Metrology, 1983: 328).

The mean is calculated for the production process, which is the essential basis for the upper and lower limits of the board, whose deviations are allowed to move within it, and this medium is related to the type of adjustment plate; It is may be expressed among the sample media $(\overline{\bar{X}})$ or the extent average $(\bar{R})$ or the average percentage of defective units $(\bar{P})$ or the average of standard deviations $(\bar{S})$. (Bishmani, 47: 2000).

Variables control panels on the production process of a commodity:

The size of production of Paramol tablets is 720 thousand tablets per shift, within 6 hours per day, with an average production rate of 120 thousand tablets / hour, which is 2000 tablets / minute, or about 44 tablets / second.

Quality control panels $(\bar{x}, S, R, x)$, it mainly depends on the selection of small samples (SUBGROUP subgroup), for Paramol tablet weight in mg. The distribution of subgroup averages is naturally distributed or approaches the natural distribution of a subgroup of 4 units or more. According to the American 
pharmacopoeias, which the company depends on in its manufacture, it allows deviations that do not exceed $5 \%$ of the target value (Target $=555.5)$, and this is what the company follows in controlling the product under study, and the specifications were as follows:

LSL $=($ Target $-5 \%$ of Target $)$

$=555.5 \mathrm{mg}-0.05(555.5)$

$=527.7 \mathrm{mg}$

CLC $=$ Target $=555.5 \mathrm{mg}$

USL $=$ (Target $+5 \%$ of Target $)$

$=555.5 \mathrm{mg}+0.05(555.5)$

$=583.3 \mathrm{mg}$

- According to the previous inspection plan, the control panels were applied ( $\mathrm{S}, \mathrm{X}, \overline{\boldsymbol{x}})$ On the current operating mode, and calculating the setting limits for each panel. The results of the application are shown in Table 2. This shows the settings for the control panel( $\left., \mathrm{S}, \mathrm{X},{ }^{\mathrm{x}}\right)$ Paramol tablet weight in $\mathrm{mg}$.

Table 2. The data of the control panels (S, X, ) for the weight of the Paramol tablet in milligrams

\begin{tabular}{|c|c|c|c|c|c|c|c|c|}
\hline \# Sample & & Sal & iples & & & $x$ & $S$ & $\mathrm{R}$ \\
\hline 1 & 557.2 & 546.7 & 568.8 & 552.2 & 570.4 & 559.06 & 10.3290 & 23.7 \\
\hline 2 & 550.3 & 543.8 & 543.9 & 558.1 & 556.6 & 550.54 & 6.7722 & 14.3 \\
\hline 3 & 538.5 & 552.9 & 540.8 & 538.0 & 546.0 & 543.24 & 6.2612 & 14.9 \\
\hline 4 & 551.2 & 552.3 & 549.4 & 547.2 & 543.0 & 548.62 & 3.6881 & 9.3 \\
\hline 5 & 553.9 & 550.4 & 547.6 & 536.2 & 553.8 & 548.38 & 7.2953 & 17.7 \\
\hline 6 & 535.6 & 544.4 & 534.1 & 545.4 & 531.5 & 538.20 & 6.2996 & 13.9 \\
\hline 7 & 550.8 & 560.3 & 558.9 & 550.4 & 559.8 & 556.04 & 4.9933 & 9.9 \\
\hline 8 & 563.1 & 548.6 & 548.1 & 548 & 556.2 & 552.80 & 6.7160 & 15.1 \\
\hline 9 & 560.8 & $581.5 \max$ & 536.9 & 556.2 & 529.6 & 553.00 & 20.5554 & 51.9 \\
\hline 10 & 539.2 & 566.2 & 543 & 543.6 & 565.9 & 551.58 & 13.3170 & 27.0 \\
\hline 11 & 538.1 & 532.4 & 546.1 & 554 & 556.8 & 545.48 & 10.3280 & 24.4 \\
\hline 12 & 558 & $\min 526.2$ & 551 & 536.2 & 538.3 & 541.94 & 12.5892 & 31.8 \\
\hline 13 & 540.6 & 537.6 & 579.2 & 531.8 & 546.9 & 547.22 & 18.6880 & 47.4 \\
\hline 14 & 541.2 & 557.8 & 565.1 & 551 & 555.5 & 554.12 & 8.8412 & 23.9 \\
\hline 15 & 542.2 & 538 & 555.5 & 540.7 & 541.2 & 543.52 & 6.8751 & 17.5 \\
\hline 16 & 544 & 534.9 & 569.5 & 561 & 568.4 & 555.56 & 15.4050 & 34.6 \\
\hline 17 & 536.2 & 573.1 & 538.1 & 533.5 & 551.2 & 546.42 & 16.3956 & 39.6 \\
\hline 18 & 544.5 & 559.6 & 576 & 547.6 & 562.4 & 558.02 & 12.6128 & 31.5 \\
\hline 19 & 544.7 & 546.8 & 542.8 & 536.9 & 528.7 & 539.98 & 7.3053 & 18.1 \\
\hline 20 & 543.5 & 548 & 567.8 & 553.2 & 533.7 & 549.24 & 12.6108 & 34.1 \\
\hline$\overline{\bar{x}}$ & 549.248 & & & & & & & \\
\hline $\bar{S}$ & 10.2639 & & & & & & & \\
\hline $\bar{R}$ & 24.53 & & & & & & & \\
\hline
\end{tabular}

From the previous table, the statistic was calculated $=549.2 \overline{\bar{x}}$ which represents the center of the sample means or subgroups and here represents the center line of the plate $\bar{x}$ the upper and lower adjustment limits or the control panel are calculated $\bar{x}$ as follows:

$$
\text { UCL }=564.4
$$

$$
L C L=534.1
$$

According to the Minitab program, the previous table data was entered, and a board was drawn $\bar{x}$ as in Figure No. (3). 


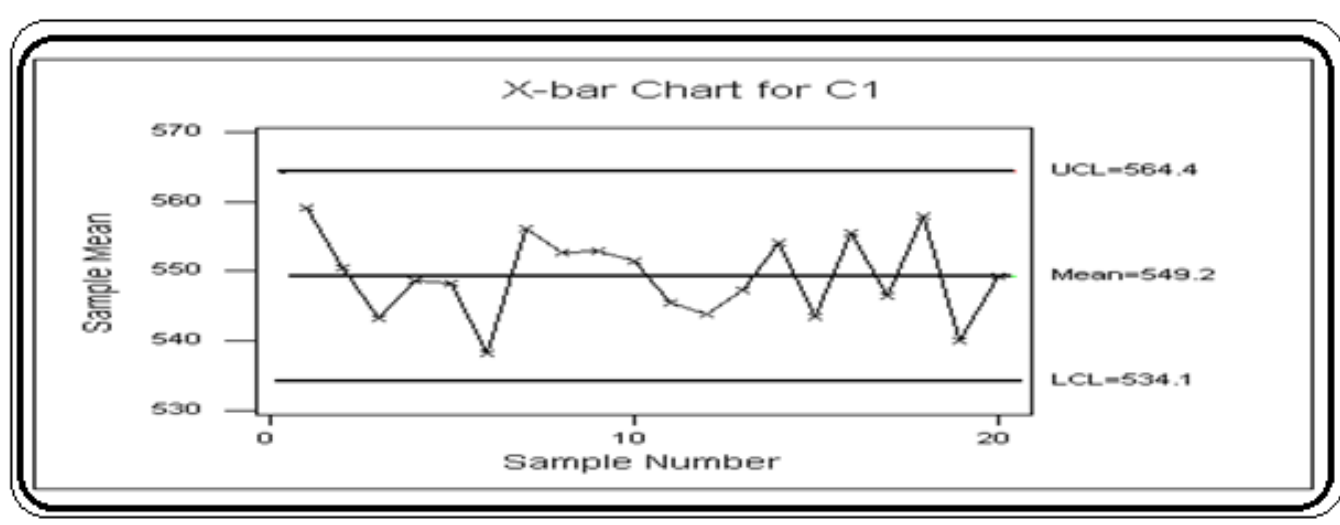

Figure 3. Illustrates a board $x$

We find from the above that, the use of probability distributions at all stages of the production process increases the efficiency and effectiveness of quality control.

This supports the validity of the hypothesis of this research.

1- The use of probability distributions maintains continuous improvement in quality and checks for convergence between inputs and outputs.

2- The use of probability distributions raises the efficiency and effectiveness of quality control.

\section{Recommendations}

Through the method used in this research, we reached:

1- The necessity of using probability distributions to control the quality of products at all stages of production.

2- Establishing periodic training programs specialized in quality control, with a focus on the use of probability distributions.

Acknowledgment: This publication was supported by Deanship of Scientific Research, Prince Sattam Bin Abdulaziz University, Alkharj, Saudi Arabia.

\section{References}

[1]. Al Daradkeh, Mamoun, and others. (2001). Total Quality Management, Safaa House for Publishing and

[2]. Distribution - Amman, First Edition

[3]. Al-Aariqi Bassim. (2000). The Effect of Applying Quality Standards on Marketing Performance. An

[4]. Applied Study on Al-Barh Cement Factory in Yemen, Master Thesis, College of Administration and

[5]. Economics, University of Kufa.

[6]. Almasna, Khaled. (2004). Statistical Control on the Quality of Cement Production in Yemen, Application

[7]. Control of Production - Application on Al-Berh Cement Factory, Master Thesis, Statistics, College of

[8]. $\quad$ Administrative Sciences, Aden University.

[9]. Al-Zamki, Fatimah. (2003). Use of Statistical Methods in Quality Control of Production - Applied Case in

[10]. Yemeni Flour Mills Company, Aden, Aden University. Master Thesis, College of Administrative

[11]. Sciences, P: 1 .

[12]. Almasna, Khaled. (2004). Statistical Control on the Quality of Cement Production in Yemen, Application 
[13]. Control of Production - Application on Al-Berh Cement Factory, Master Thesis, Statistics, College of

[14]. Administrative Sciences, Aden University.

[15]. Bashmani, Shakeb. (2000). Statistical Techniques in Quality Control of Industrial Products, University of

[16]. Aleppo, P: 95

[17]. George, Doon. (2004). Statistic for Traders, New Entrance, Translation of Sultan Abdul Hamid, Dar-el-

[18]. Mirreekh P: 36

[19]. Hongyi Sun. Total quality management, ISO 9000 certification and per formance improvement,

[20]. International Journal of quality \& Reliability Management Vol. 17 (No. 2, 2000).

[21]. Mohammed, Hala. (2015). Use of Statistical Sampling Plans by Attributes in Quality Control of Industrial

[22]. Product, Master Thesis, Damascus University, Syria.

[23]. Montgomery, D.C. (2009). Introduction to Statistical Quality Control 6th ed., John Wiley \& Sons.

[24]. Peter Knutron, A model approach to self - assessment, Works Management, Vol .47 (No. 12, 1994).

[25]. Robert F L ynch, Thomas J. Werner and Livia C.Lynch. (1992). continuous improvement: Teams and Tools,

[26]. Atlanta: Gual team, Inc.

[27]. Weimer, Richard C. ( 1993 ). Statistics, (2nd. ed), W.m.c Brown Publisher, U.S.A. 